\title{
Long non-coding RNA DANCR promotes nasopharyngeal carcinoma cell proliferation and migration
}

\author{
YALIN HAO*, HUI ZHAO*, XIAOJIE JIN, PING HE, JIAFENG ZHANG, \\ QIONGNA DONG, WEIWEI SHI and MIAOMIAO ZHAO \\ Department of Otorhinolaryngology, Ren Ji Hospital, School of Medicine, \\ Shanghai Jiaotong University, Shanghai 201112, P.R. China
}

Received May 10, 2018; Accepted January 16, 2019

DOI: $10.3892 / \mathrm{mmr} .2019 .9906$

\begin{abstract}
Aberrant expression of numerous long non-coding RNAs (lncRNAs) has been reported to be associated with nasopharyngeal carcinoma (NPC). The present study aimed to investigate the expression and function of lncRNA differentiation antagonizing non-protein coding RNA (DANCR) in NPC pathogenesis. Reverse transcription-quantitative polymerase chain reaction results suggested that DANCR was significantly upregulated in NPC cells. Overexpression of DANCR promoted $5-8 \mathrm{~F}$ cell proliferation and migration, as detected by Cell Counting Kit-8, colony formation and wound healing assays. DANCR was additionally identified to inhibit apoptosis, as determined by flow cytometric analysis. Furthermore, DANCR knockdown suppressed cell proliferation and migration, and promoted cell apoptosis in SUNE-1 cell. Western blot analysis suggested that DANCR regulated the phosphorylation of AKT serine/threonine kinase and the protein expression of PTEN in NPC cells. Knockdown of DANCR decreased tumor growth in a xenograft model following subcutaneous injection of SUNE-1 cells. Collectively, the present results suggested that DANCR regulated the proliferation, migration and apoptosis of NPC cells.
\end{abstract}

\section{Introduction}

Nasopharyngeal carcinoma (NPC) is a malignant tumor of the head and neck with a variable incidence rate according to race, ethnicity and geographical location; particularly high rates have been identified in the populations of South China

Correspondence to: Professor Xiaojie Jin, Department of Otorhinolaryngology, South Campus, Ren Ji Hospital, School of Medicine, Shanghai Jiaotong University, 2000 Jiangyue Road, Shanghai 201112, P.R. China

E-mail: jinxiaojie2@126.com

${ }^{*}$ Contributed equally

Key words: differentiation antagonizing non-protein coding RNA, nasopharyngeal carcinoma, migration, apoptosis, protein kinase $\mathrm{B}$ and Southeast Asia (1). A previous study demonstrated that Epstein-Barr virus infection, genetic susceptibility and environmental factors are the principal risk factors associated with the pathogenesis of NPC (2). NPC is characterized by high rates of local and distant metastasis in early stages. Although the combination of radiotherapy and chemotherapy has improved NPC outcomes, survival rates remain poor (3). Therefore, the characterization of the molecular mechanisms underlying NPC development has attracted increasing attention from researchers.

Long non-coding RNAs (lncRNAs) are non-coding RNAs $\geq 200$ nucleotides in length that regulate gene expression via various mechanisms, including the modulation of RNA stability, the translation rates of mRNA and the regulation of the epigenetic machinery (4). Aberrant expression of lncRNAs is associated with a number of malignancies. Furthermore, various lncRNAs have been demonstrated to serve as oncogenes or tumor suppressor genes, regulating cancer cell proliferation, migration, apoptosis and chemotherapy resistance (5). Previous studies have demonstrated that numerous lncRNAs, including small nucleolar RNA host gene 1 and nuclear paraspeckle assembly transcript 1 , are dysregulated in NPC tissues, serving a role in cancer progression $(6,7)$.

The lncRNA differentiation antagonizing non-protein coding RNA (DANCR) was identified to be associated with the dedifferentiation of epidermal cells (8). Additionally, DANCR has been demonstrated to be involved in tumorigenesis in various types of cancer, including colorectal cancer, breast cancer and lung adenocarcinoma, by regulating protein expression or by sponging microRNAs (9-11). Wen et al (12) observed that DANCR expression was increased in metastatic NPC cells, suggesting a role for DANCR in NPC development. However, the molecular mechanism underlying DANCR in NPC remains unclear. In the present study, DANCR expression levels were measured in NPC cells, and the effect of DANCR on the proliferation, migration and apoptosis of NPC cells was investigated using loss- and gain-of-function models. Furthermore, DANCR was identified to regulate the protein expression levels of AKT serine/threonine kinase $(\mathrm{AKT})$, phosphatase and tensin homolog (PTEN), and a number of apoptosis-associated factors, including BCL2 apoptosis regulator (BCL2) and BCL2 associated X apoptosis regulator $(\mathrm{BAX})$. 


\section{Materials and methods}

Cell lines and transfection. The human NPC cell lines 5-8F, SUNE-1, C666-1 and the normal human nasopharyngeal epithelial cell line NP69 (Sun Yat-sen University, Guangzhou, China) were maintained in our laboratory following authentication. RPMI-1640 medium (Gibco; Thermo Fisher Scientific, Inc., Waltham, MA, USA) supplemented with $10 \%$ fetal bovine serum (Gibco; Thermo Fisher Scientific, Inc.) was used to culture NPC cell lines in $5 \% \mathrm{CO}_{2}$ at $37^{\circ} \mathrm{C}$. Keratinocyte serum-free medium with epidermal growth factor (Invitrogen; Thermo Fisher Scientific, Inc.) was used to culture NP69 cells in $5 \% \mathrm{CO}_{2}$ at $37^{\circ} \mathrm{C}$.

Small interfering RNA targeting DANCR (si-DANCR) and scrambled negative control (si-NC) were purchased from Shanghai GenePharma Co., Ltd. (Shanghai, China). Knockdown was performed using the following siRNAs: si-DANCR: 5'-GCUGGUAUUUCAAUUGACU-3'; si-NC: 5'-UUCUCC GAACGUGUCACGU-3'. DANCR overexpression plasmid (pcDNA-DANCR) was constructed by cloning DANCR into a pcDNA vector (Invitrogen; Thermo Fisher Scientific, Inc.). To amplify the coding sequence of DANCR, the cDNA from SUNE-1 cells was used as a template, and the reaction was performed using the PrimeSTAR HS DNA polymerase (Takara Biotechnology Co., Ltd., Dalian, China) with the following primers: Forward (F): 5'-CGGGGTACCGCCCTTGCCCAG AGTCTTCCCGG-3' and reverse (R): 5'-CCGCTCGAGGTC AGGCCAAGTAAGTTTATTAACCT-3'. The thermocycling conditions were as follows: Initial denaturation at $95^{\circ} \mathrm{C}$ for $5 \mathrm{~min}$, followed by 30 cycles at $95^{\circ} \mathrm{C}$ for $1 \mathrm{~min}, 50^{\circ} \mathrm{C}$ for $2 \mathrm{~min}$, $72^{\circ} \mathrm{C}$ for $2 \mathrm{~min}$ and a final extension at $72^{\circ} \mathrm{C}$ for $10 \mathrm{~min}$. SUNE-1 cells and $5-8 \mathrm{~F}$ cells at $40-70 \%$ confluence were transfected with $500 \mathrm{ng}$ plasmid or with si-DANCR/si-NC at a concentration of $50 \mathrm{nM}$ using Lipofectamine ${ }^{\circledR} 2000$ (Invitrogen; Thermo Fisher Scientific, Inc.). Following incubation for 48 h, cells were harvested for further experimentation.

Reverse transcription-quantitative polymerase chain reaction $(R T-q P C R)$. Total RNA was isolated from NPC cells, NP69 cells and tumor tissues using TRIzol ${ }^{\circledR}$ reagent (Invitrogen; Thermo Fisher Scientific, Inc.), according to the manufacturer's protocol. A total of $1 \mathrm{ml}$ TRIzol ${ }^{\circledR}$ was incubated with the cell suspensions or tissues powder $(100 \mathrm{mg})$ for $5 \mathrm{~min}$ at room temperature, subsequently $0.2 \mathrm{ml}$ chloroform was added and incubated for $10 \mathrm{~min}$ at room temperature. Following centrifugation $\left(12,000 \mathrm{x} \mathrm{g}, 15 \mathrm{~min}, 4^{\circ} \mathrm{C}\right)$, the supernatant was precipitated by alcohol and centrifuged $\left(7,500 \mathrm{xg}, 5 \mathrm{~min}, 4^{\circ} \mathrm{C}\right)$. RT was performed using M-MLV reverse transcriptase (Takara Biotechnology Co., Ltd.) with $2.5 \mu \mathrm{M}$ primers, $5 \mathrm{X}$ First-Strand Buffer, dNTP Mix and DTT (Takara Biotechnology Co., Ltd.). The temperature protocol was as follows: $70^{\circ} \mathrm{C}$ for $3 \mathrm{~min}, 42^{\circ} \mathrm{C}$ for $60 \mathrm{~min}$ and $70^{\circ} \mathrm{C}$ for $15 \mathrm{~min}$. qPCR was conducted using a SYBR Green kit (Takara Biotechnology Co., Ltd.) and the 7500 Real-Time PCR System (Applied Biosystems; Thermo Fisher Scientific, Inc.) (13). The thermocycling conditions were as follows: Initial denaturation at $95^{\circ} \mathrm{C}$ for $30 \mathrm{sec}$, followed by 40 cycles at $95^{\circ} \mathrm{C}$ for $5 \mathrm{sec}$ and at $60^{\circ} \mathrm{C}$ for $40 \mathrm{sec}$. The qPCR primers used were as follows: DANCR, forward 5'-CGTACT AACTTGTAGCAACC-3', reverse 5'-TCAGCTGCATTGAGT TAGCG-3'; and GAPDH, forward 5'-ACTCACTCAAGATTG

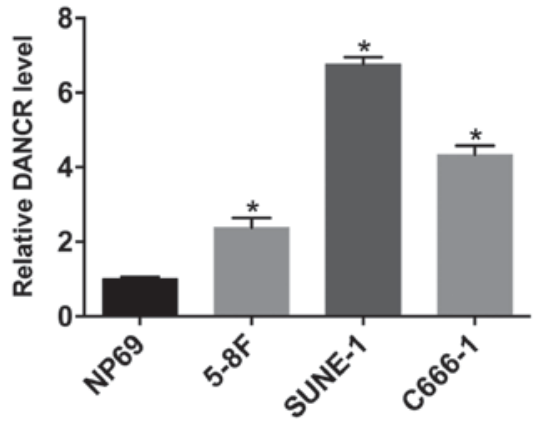

Figure 1. DANCR expression in vitro. DANCR expression level was measured in the nasopharyngeal carcinoma cell lines 5-8F, SUNE-1, C666-1 and the normal human nasopharyngeal epithelial cell line NP69. ${ }^{*} \mathrm{P}<0.05$ vs. NP69 cells. DANCR, differentiation antagonizing non-protein coding RNA.

TCAGCA-3', reverse 5'-GGCCATCACGCCACAGCTTT-3'). Each experiment was performed three times, and relative gene expression was determined using the $2^{-\Delta \Delta \mathrm{Cq}}$ quantification method (14).

Western blot analysis. Total protein was extracted from 5-8F cells and SUNE-1 cells using Pierce cell lysis buffer (Pierce; Thermo Fisher Scientific, Inc.). Protein concentration was quantified using a bicinchoninic acid assay kit (Takara Biotechnology Co., Ltd.). A total of $50 \mu \mathrm{g}$ protein was loaded per lane. Proteins were separated by $10 \%$ SDS-PAGE and transferred onto polyvinylidene difluoride membranes (Merck KGaA, Darmstadt, Germany). Following blocking in 5\% skim milk for $2 \mathrm{~h}$ at $37^{\circ} \mathrm{C}$, the membranes were incubated overnight at $4^{\circ} \mathrm{C}$ with the following primary antibodies: Phosphorylated (p)-pan-AKT (1:1,000; cat. no. ab38449; Abcam, Cambridge, UK), PTEN (1:1,000; cat. no. ab31392; Abcam), BCL2 (1:1,000; cat. no. ab196495; Abcam), BAX (1:1,000; ab53154; Abcam) and $\beta$-actin (1:2,000; cat. no. ab8227; Abcam). Subsequently, membranes were incubated with the appropriate horseradish peroxidase-conjugated secondary antibody (1:2,000; cat.no. ab6728; Abcam) for $2 \mathrm{~h}$ at room temperature. Immunoreactive bands were visualized with an enhanced chemiluminescence detection system (Merck KGaA). Relative amounts of proteins were quantified by absorbance analysis using ImageJ software (version 1.8.0; National Institutes of Health, Bethesda, MD, USA), and the level was normalized to $\beta$-actin.

Cell proliferation assay. A total of $2 \times 10^{3}$ cells/well of transfected cells were seeded into 96-well plates and cultured at $37^{\circ} \mathrm{C}$ for $0,24,48$ and $72 \mathrm{~h}$. Subsequently, $100 \mu \mathrm{l}$ Cell Counting Kit-8 (CCK-8; Dojindo Molecular Technologies, Inc., Kumamoto, Japan) reagent was added, and cells were incubated with CCK-8 reagent for $4 \mathrm{~h}$. Absorbance at $450 \mathrm{~nm}$ was measured using a microplate reader (Thermo Fisher Scientific, Inc.).

Colony formation assay. Transfected cells $\left(1 \times 10^{3}\right.$ cells/well $)$ cultured in complete medium were seeded in 6-well plates. Following 14 days, colonies were fixed in $100 \%$ methanol for $15 \mathrm{~min}$ at room temperature. The fixing solution was removed and $10 \%$ Giemsa stain solution was used to stain the colonies 
A

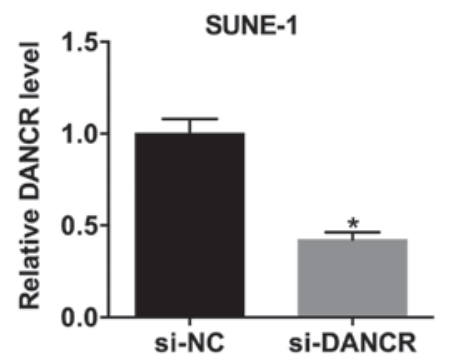

C

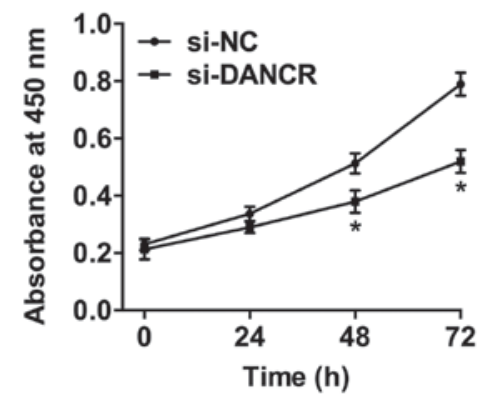

B
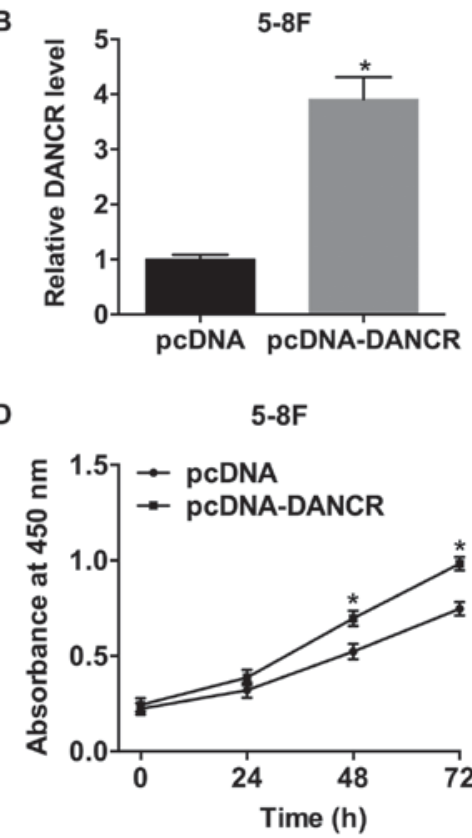

Figure 2. DANCR promotes the viability of nasopharyngeal carcinoma cells. DANCR expression level in (A) SUNE-1 cells transfected with si-DANCR and (B) 5-8F cells transfected with pcDNA-DANCR. Viability of (C) SUNE-1 cells transfected with si-DANCR and (D) 5-8F cells transfected with pcDNA-DANCR. ${ }^{*} \mathrm{P}<0.05$ vs. respective control. DANCR, differentiation antagonizing non-protein coding RNA; NC, negative control; si-, small interfering RNA.

for $30 \mathrm{~min}$ at room temperature. The plates were subsequently imaged under a light microscope (BX51; Olympus Corporation, Tokyo, Japan; magnification, x100) and colonies were counted.

Wound healing assay. A total of $5 \times 10^{5}$ cells cultured in serum-free RPMI-1640 medium, to limit proliferation, were seeded in 6-well plates. Subsequently, a scratch was made through the single cell layer using a $10 \mu \mathrm{l}$ pipette tip, and the cells were washed with PBS. The cells were incubated at $37^{\circ} \mathrm{C}$ for $48 \mathrm{~h}$ and subsequently imaged using an inverted fluorescence microscope (IX71; Olympus Corporation; magnification, x100). Each experiment was performed in triplicate.

Flow cytometric apoptosis analysis. SUNE-1 and 5-8F cells were transfected with si-DANCR and pcDNA-DANCR, respectively. Following transfection and a subsequent incubation of $48 \mathrm{~h}$, cells were harvested and incubated with $5 \mu \mathrm{l}$ Annexin V-fluorescein isothiocyanate (FITC) for $20 \mathrm{~min}$, and subsequently with $10 \mu \mathrm{l}$ propidium iodide (PI) for $10 \mathrm{~min}$ (FITC/PI kit; R\&D Systems, Inc., Minneapolis, MN, USA). Apoptotic cells were observed and analyzed using a flow cytometer (Becton, Dickinson and Company, Franklin Lakes, NJ, USA) and FlowJo software (version 7; Tree Star, Inc., Ashland, OR, USA). All experiments were performed in triplicate.

NPC xenograft mouse model. A total of 12 male BALB/c $\mathrm{c}^{\text {nu/nu }}$ mice (weight, 25-30 g; age, 6-weeks) purchased from Beijing Vital River Laboratory Animal Technology Co., Ltd. (Beijing, China) were maintained under a 12-h light/dark cycle at $20-22^{\circ} \mathrm{C}$ and $40-60 \%$ relative humidity with access to food and water ad libitum. The lentiviral vectors (LV) containing a short hairpin RNA targeting DANCR (sh-DANCR) and the LV-control (ctrl) were purchased from Guangzhou RiboBio
Co., Ltd. (Guangzhou, China) and transduced into SUNE-1 cells with a multiplicity of infection of 50. Following a 72-h incubation, the cells $\left(2 \times 10^{6}\right)$ were injected into the right flanks of nude mice. A total of six mice were used in each group. Tumor size was measured every 7 days for 5 weeks. Tumor volumes $\left(\mathrm{mm}^{3}\right)$ were calculated using the following formula: Volume $=0.5 \mathrm{x}$ length $\mathrm{x}$ width $^{2}$. All animal experiments were approved by The Institutional Animal Care and Use Committee of Shanghai Jiaotong University (Shanghai, China).

Statistical analysis. Data are presented as the mean \pm standard deviation from three replicates. Statistical differences were assessed using Student's t-test or one-way analysis of variance followed by Student-Newman-Keuls post hoc test for multiple comparisons. Statistical analysis was conducted using SPSS software (version 23; IBM Corp., Armonk, NY, USA). P<0.05 was considered to indicate a statistically significant difference.

\section{Results}

DANCR is upregulated in NPC cells. To investigate DANCR expression in NPC cells, RT-qPCR was performed in NPC and control NP69 cells. DANCR was significantly upregulated in NPC cells compared with NP69 cells (Fig. 1). Among the NPC cell lines examined, SUNE-1 and 5-8F cells exhibited the highest and lowest DANCR expression levels, respectively.

DANCR increases the viability of NPC cells. SUNE-1 cells were transfected with si-DANCR to knockdown DANCR and 5-8F cells were transfected with pcDNA-DANCR to overexpress DANCR. si-DANCR dramatically decreased DANCR expression in SUNE-1 cells, and DANCR was significantly upregulated in 5-8F cells transfected with pcDNA-DANCR (Fig. 2A and B). Silencing DANCR significantly inhibited the 
A

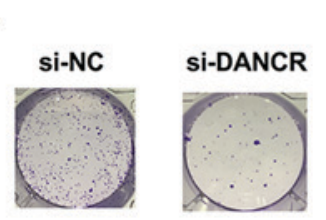

C

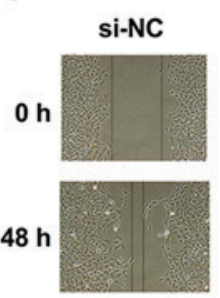

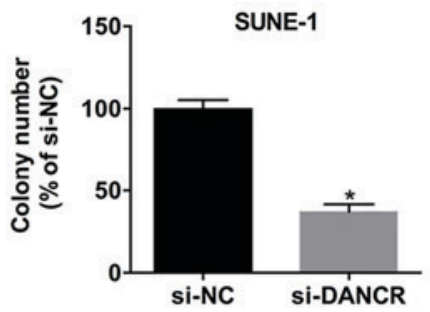

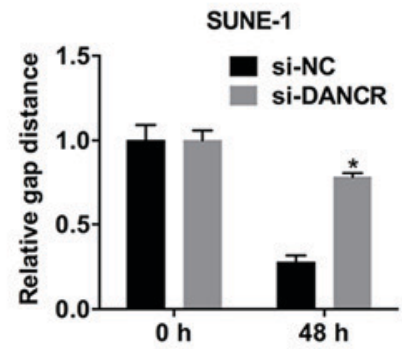

B
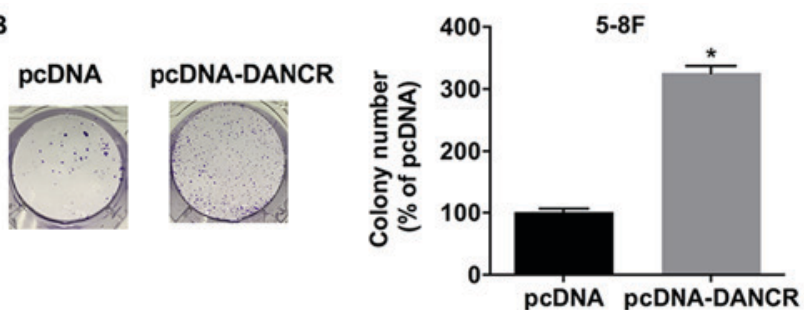

D

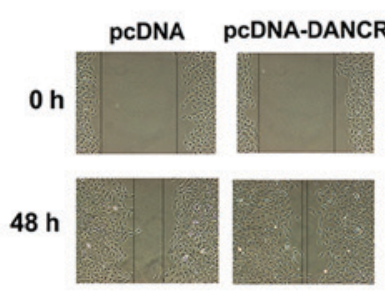

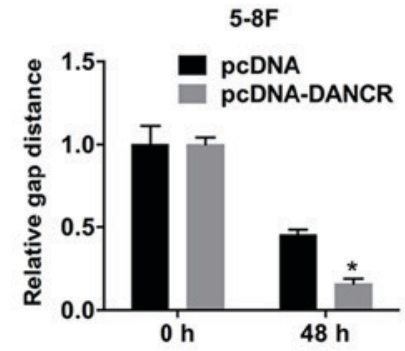

Figure 3. DANCR promotes colony formation and cell migration in nasopharyngeal carcinoma cells. (A) Silencing DANCR suppressed the colony formation in SUNE-1 cells. (B) Colony formation was increased in DANCR-overexpressing 5-8F cells. Wound healing assays were performed to detect the migratory abilities of (C) SUNE-1 cells transfected with si-DANCR and (D) 5-8F cells transfected with pcDNA-DANCR. "P<0.05 vs. respective control. si-, small interfering RNA; NC, negative control; DANCR, differentiation antagonizing non-protein coding RNA.

A

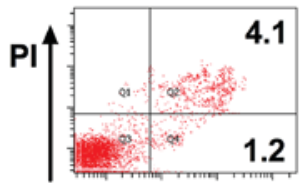

2

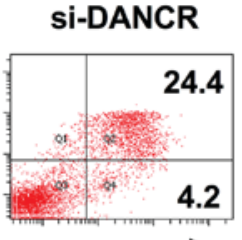

Annexin V

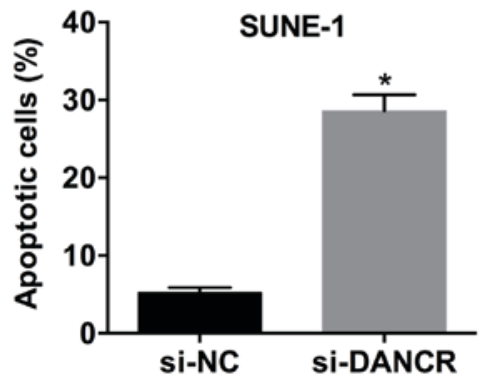

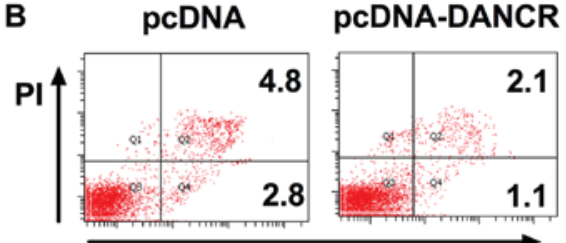

Annexin V

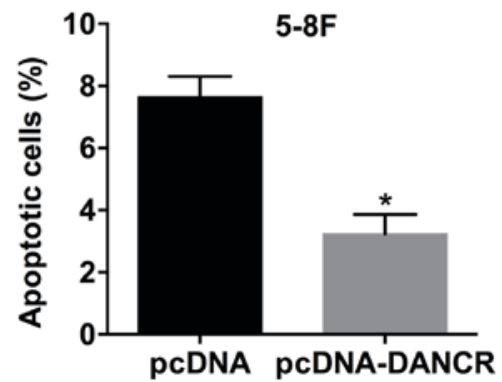

Figure 4. DANCR suppresses apoptosis in nasopharyngeal carcinoma cells. Apoptosis rates were analyzed in (A) SUNE-1 cells transfected with si-DANCR and (B) 5-8F cells transfected with pcDNA-DANCR. "P $<0.05$ vs. respective control. si-, small interfering RNA; NC, negative control; DANCR, differentiation antagonizing non-protein coding RNA; PI, propidium iodide.

viability of SUNE-1 cells (Fig. 2C). Bu contrast, compared with the negative control, the viability of cells transfected with pcDNA-DANCR was significantly increased (Fig. 2D).

DANCR promotes colony formation and migration in NPC cells. Silencing DANCR suppressed colony formation in SUNE-1 cells, whereas, DANCR overexpression significantly increased colony formation in 5-8F cells (Fig. 3A and B). The migration of SUNE-1 cells transfected with si-DANCR was significantly decreased compared with si-NC (Fig. 3C), whereas, overexpressing DANCR significantly increased the migratory ability of 5-8F cells (Fig. 3D).

DANCR overexpression suppresses apoptosis in NPC cells. The apoptosis rate of SUNE-1 cells transfected with si-DANCR was significantly increased compared with si-NC (Fig. 4A). Additionally, DANCR overexpression decreased the apoptosis rate of 5-8F cells (Fig. 4B). The present results suggested that DANCR suppressed apoptosis in NPC cells.

DANCR alters the protein expression level of AKT, PTEN and apoptosis-associated factors. To investigate the mechanism of DANCR activity in NPC cell phenotypes, the effect of DANCR on the phosphorylation of AKT, and protein expression levels of PTEN and the apoptosis-associated factors BAX and BCL2 was examined. Western blot analysis suggested that silencing DANCR decreased the phosphorylation of AKT, but increased total PTEN levels in SUNE-1 cells (Fig. 5A). Overexpression of DANCR in $5-8 \mathrm{~F}$ cells resulted in the opposite effects (Fig. 5B). Following DANCR knockdown, 
A

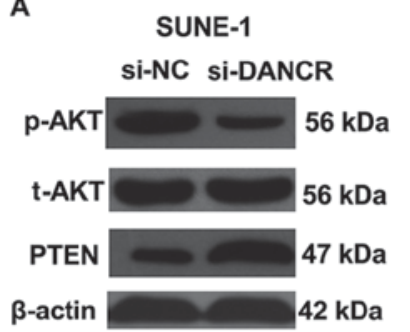

C

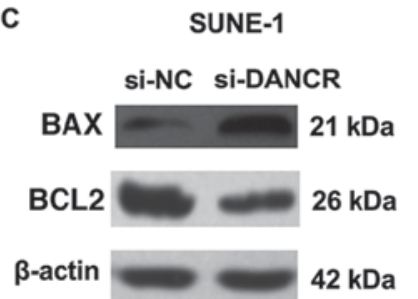

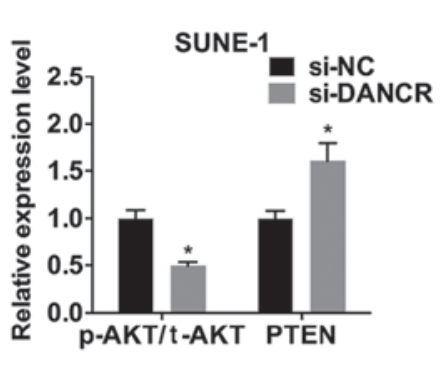

B
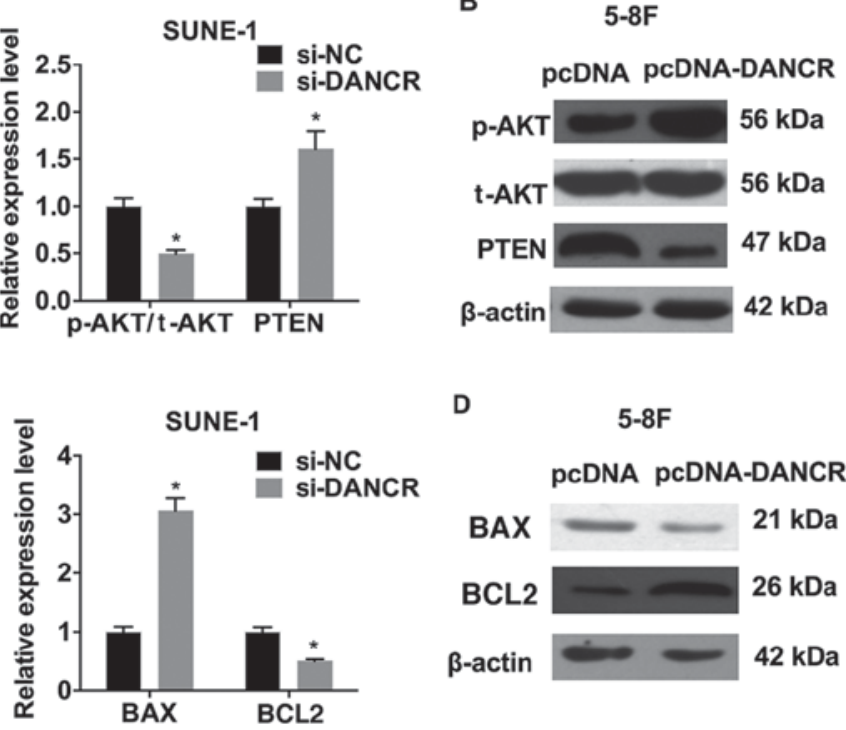

D

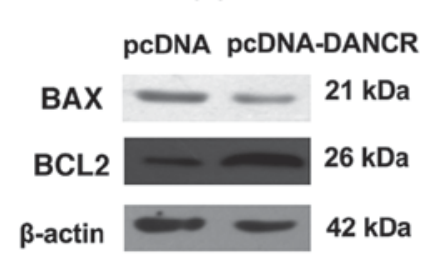

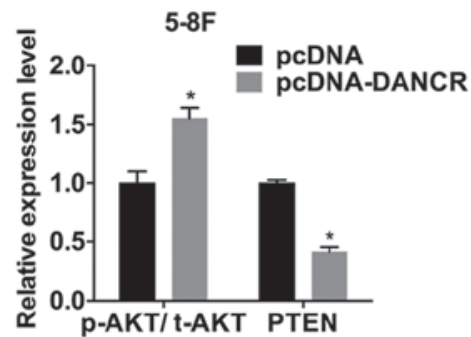

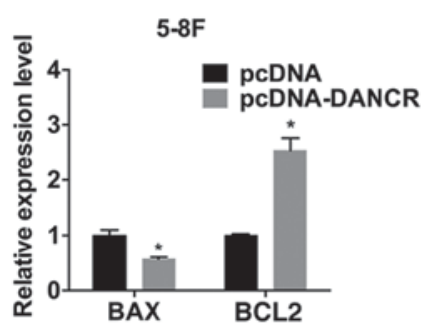

Figure 5. DANCR regulates the protein expression levels of AKT, PTEN and apoptosis-associated factors. AKT and PTEN were detected by western blot analysis in (A) SUNE-1 cells transfected with si-DANCR and (B) 5-8F cells transfected with pcDNA-DANCR. BAX and BCL2 protein levels were examined in (C) SUNE-1 cells transfected with si-DANCR and (D) 5-8F cells transfected with pcDNA-DANCR. "P<0.05 vs. respective control. si-, small interfering RNA; NC, negative control; DANCR, differentiation antagonizing non-protein coding RNA; p-, phosphorylated; AKT, AKT serine/threonine kinase; t-, total; PTEN, phosphatase and tensin homolog; BCL2, BCL2, apoptosis regulator; BAX, BCL2 associated X, apoptosis regulator.

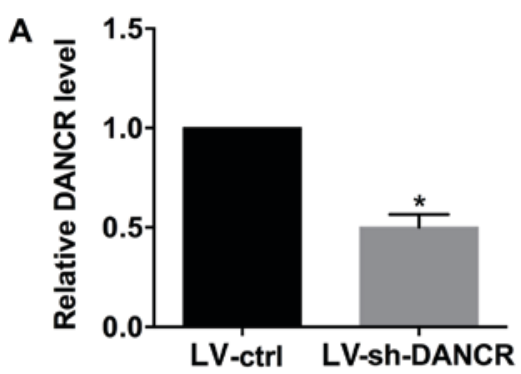

c

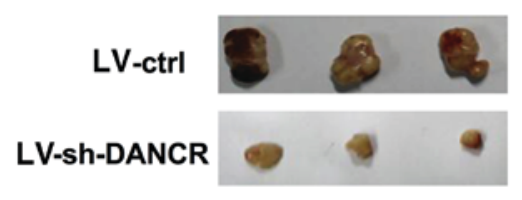

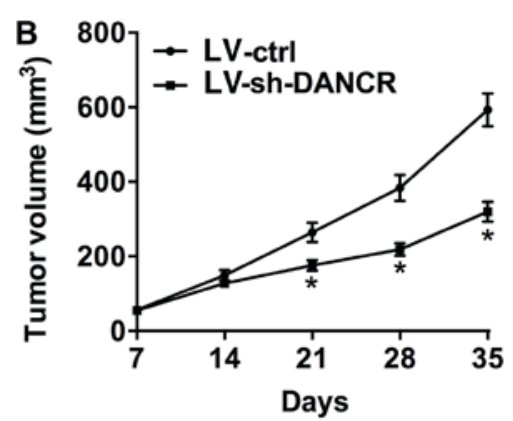

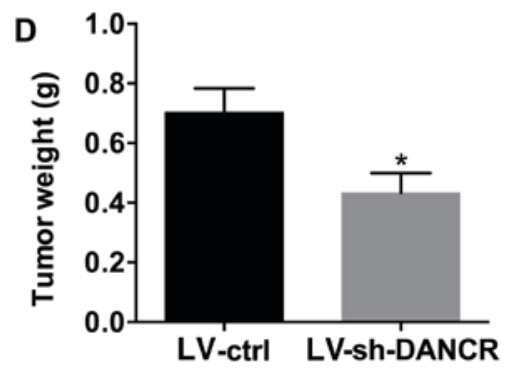

Figure 6. DANCR promotes tumor growth in vivo. (A) DANCR expression in tumor tissue. (B) Tumor volumes were measured every 7 days. (C) Representative images of tumors 35 days following subcutaneous cell injection. (D) Tumor weight measurement at 35 days. $\mathrm{P}<0.05$ vs. LV-ctrl group. DANCR, differentiation antagonizing non-protein coding RNA; LV-, lentiviral vector; ctrl, control; sh-, short hairpin RNA.

BAX and BCL2 protein expression levels were significantly increased and decreased, respectively (Fig. 5C). Conversely, DANCR overexpression produced the opposite results in $5-8 \mathrm{~F}$ cells (Fig. 5D).

DANCR promotes tumor growth in vivo. Murine xenograft models were established by injecting SUNE-1 cells transduced with LV-ctrl or LV-sh-DANCR into nude mice. Subsequently, the tumor size was measured every 7 days for 5 weeks. DANCR was significantly downregulated in the tumor tissues derived from SUNE-1 cells transduced with LV-sh-DANCR compared with LV-ctrl (Fig. 6A). The xenografts results suggested that LV-sh-DANCR was able to inhibit tumor growth in vivo (Fig. 6B-D).

\section{Discussion}

lncRNAs serve critical roles in NPC progression and are associated with cancer prognosis. Ma et al (15) identified that the lncRNA HOX transcript antisense RNA serves as an 
oncogene in NPC progression and recurrence by upregulating fatty acid synthase. NPTN intronic transcript 1 was identified to be repressed by enhancer of zeste 2 polycomb repressive complex 2 subunit and to serve tumor suppressive roles in NPC (16). Additionally, oncogenic functions of HNF1A antisense RNA 1 have been identified in NPC, and this lncRNA was demonstrated to be involved in epithelial-mesenchymal transition (17). Although numerous IncRNAs have been reported to be involved in NPC progression, a complete understanding of the lncRNAs involved in this type of malignancy is required to develop effective therapeutic strategies to treat NPC.

In the present study, it was identified that DANCR expression was significantly increased in NPC cells. Gain-of-function experiments suggested that DANCR overexpression promoted cell proliferation and migration, and inhibited apoptosis in 5-8F cells. By contrast, silencing DANCR in SUNE-1 cells suppressed their proliferation and migration, and increased apoptosis. Furthermore, knockdown of DANCR decreased tumor xenograft growth in vivo.

DANCR was identified to suppress progenitor differentiation in epidermal cells (8). Additionally, DANCR may serve as an oncogene in various types of cancer, including osteosarcoma, gastric cancer and colorectal cancer $(9,18,19)$. Notably, DANCR was identified to serve as a tumor suppressor in others malignancies, including breast cancer and renal cell carcinoma $(11,20)$. DANCR is involved in the development of numerous diseases via various mechanisms. DANCR serves as a competing endogenous RNA for microRNA (miR)-577 in colorectal cancer and for miR-33a-5p in osteosarcoma $(9,19)$. In prostate cancer, DANCR promotes cell invasion by epigenetically suppressing tissue inhibitor of metalloproteinase 2 and 3 (21).

To examine the function of DANCR in NPC, the mechanism underlying the regulation of AKT phosphorylation and the protein expression levels of PTEN by DANCR was investigated. The present results suggested that silencing DANCR decreased the phosphorylation AKT and increased the protein expression level of PTEN in SUNE-1 cells; whereas, overexpression of DANCR led to the opposite effects in $5-8 \mathrm{~F}$ cells. PTEN is a tumor suppressor in numerous types of cancer (22). A previous study suggested that the expression level of PTEN was decreased in NPC, indicating a tumor suppressive role for this lncRNA (23). PTEN negatively regulates the phosphatidylinositol 3-kinase (PI3K)/AKT pathway, which is associated with proliferation, migration, invasion and inhibition of apoptosis during cancer development (24). Downregulation of PTEN was demonstrated to activate the PI3K/AKT pathway in NPC (25). In the present study, the effect of DANCR on the protein expression levels of the apoptosis-associated factors BAX and BCL2 was additionally investigated. Following DANCR knockdown, BAX and BCL2 protein expression levels were increased and decreased, respectively. By contrast, DANCR overexpression downregulated BAX expression and upregulated BCL2 expression in $5-8 \mathrm{~F}$ cells. BAX and BCL2 are proteins associated with apoptosis. BAX is a pro-apoptotic protein, whereas, BCL2 is an anti-apoptotic factor (26). DANCR may be involved in cancer progression via various processes, including the regulation of mRNA stability and the alteration of epigenetic marks, and may serve as a competing endogenous RNA for multiple miRNAs. Although in the present study it was identified that DANCR regulated the phosphorylation of AKT, and the expression of PTEN and apoptosis-associated factors, DANCR may regulate the expression of multiple genes in NPC, and further studies are required to investigate this possibility.

The present study identified that the IncRNA DANCR was upregulated in NPC cells. DANCR promoted cell proliferation and migration, and inhibited apoptosis in NPC cells. Mechanistically, DANCR regulated the levels of p-AKT, PTEN, BAX and BCL2 in NPC cells. The present results suggested that DANCR may represent a promising therapeutic target for the treatment of NPC.

\section{Acknowledgements}

Not applicable.

\section{Funding}

The present study was supported by The Science Identification of Shanghai (grant no. 2016034A; China).

\section{Availability of data and materials}

The datasets used and/or analyzed during the current study are available from the corresponding author on reasonable request.

\section{Authors' contributions}

$\mathrm{YH}, \mathrm{HZ}$ and $\mathrm{XJ}$ designed the study. $\mathrm{YH}$ performed the experiments and wrote the paper. HZ performed the statistical analyses. PH, JZ, QD, WS and MZ contributed in performing the cell and animal experiments. All authors read and approved the final manuscript.

\section{Ethics approval and consent to participate}

The present study was approved by The Clinical Research Ethics Committee of Shanghai Jiaotong University (Shanghai, China).

\section{Patient consent for publication}

Not applicable.

\section{Competing interests}

The authors declare that they have no competing interests.

\section{References}

1. Katano A, Takahashi W, Yamashita H, Yamamoto K, Ando M, Yoshida M, Saito Y, Abe O and Nakagawa K: Radiotherapy alone and with concurrent chemotherapy for nasopharyngeal carcinoma: A retrospective study. Medicine (Baltimore) 97: e0502, 2018.

2. Zhang Y, Wang H, Liu Y, Wang C, Wang J, Long C, Guo W and Sun X: Baicalein inhibits growth of Epstein-Barr virus-positive nasopharyngeal carcinoma by repressing the activity of EBNA1 Q-promoter. Biomed Pharmacother 102: 1003-1014, 2018. 
3. Lee AW, Ma BB, Ng WT and Chan AT: Management of nasopharyngeal carcinoma: Current practice and future perspective. J Clin Oncol 33: 3356-3364, 2015.

4. Hu G, Niu F, Humburg BA, Liao K, Bendi S, Callen S, Fox HS and Buch S: Molecular mechanisms of long noncoding RNAs and their role in disease pathogenesis. Oncotarget 9: 18648-18663, 2018.

5. Huang G, Jiang H, Lin Y, Wu Y, Cai W, Shi B, Luo Y, Jian Z and Zhou X: lncAKHE enhances cell growth and migration in hepatocellular carcinoma via activation of $\mathrm{NOTCH} 2$ signaling. Cell Death Dis 9: 487, 2018.

6. Liu F, Tai Y and Ma J: lncRNA NEAT1/let-7a-5p axis regulates the cisplatin resistance in nasopharyngeal carcinoma by targeting Rsf-1 and modulating the Ras-MAPK pathway. Cancer Biol Ther 19: 534-542, 2018.

7. Lan $X$ and Liu X: lncRNA SNHG1 functions as a ceRNA to antagonize the effect of miR-145a-5p on the down-regulation of NUAK1 in nasopharyngeal carcinoma cell. J Cell Mol Med: Mar 25, 2018 (Epub ahead of print). doi: 10.1111/jcmm.13497.

8. Kretz M, Webster DE, Flockhart RJ, Lee CS, Zehnder A Lopez-Pajares V, Qu K, Zheng GX, Chow J, Kim GE, et al: Suppression of progenitor differentiation requires the long noncoding RNA ANCR. Genes Dev 26: 338-343, 2012.

9. Wang Y, Lu Z, Wang N, Feng J, Zhang J, Luan L, Zhao W and Zeng X: Long noncoding RNA DANCR promotes colorecta cancer proliferation and metastasis via miR-577 sponging. Exp Mol Med 50: 57, 2018.

10. Zhen Q, Gao LN, Wang RF, Chu WW, Zhang YX, Zhao XJ, Lv BL and Liu JB: IncRNA DANCR promotes lung cancer by sequestering miR-216a. Cancer Control 25: 1073274818769849 , 2018.

11. Sha S, Yuan D, Liu Y, Han B and Zhong N: Targeting long non-coding RNA DANCR inhibits triple negative breast cancer progression. Biol Open 6: 1310-1316, 2017.

12. Wen X, Tang X, Li Y, Ren X, He Q, Yang X, Zhang J, Wang Y, Ma $J$ and Liu N: Microarray expression profiling of long non-coding RNAs involved in nasopharyngeal carcinoma metastasis. Int J Mol Sci 17: E1956, 2016.

13. Xu SY, Huang X and Cheong KL: Recent advances in marine algae polysaccharides: Isolation, structure, and activities. Mar Drugs 15: E388, 2017.

14. Livak KJ and Schmittgen TD: Analysis of relative gene expression data using real-time quantitative PCR and the 2(-Delta Delta C(T)) method. Methods 25: 402-408, 2001.
15. Ma DD, Yuan LL and Lin LQ: lncRNA HOTAIR contributes to the tumorigenesis of nasopharyngeal carcinoma via up-regulating FASN. Eur Rev Med Pharmacol Sci 21: 5143-5152, 2017.

16. Sun Q, Liu H, Li L, Zhang S, Liu K, Liu Y and Yang C: Long noncoding RNA-LET, which is repressed by EZH2, inhibits cell proliferation and induces apoptosis of nasopharyngeal carcinoma cell. Med Oncol 32: 226, 2015.

17. Zhuang K, Wu Q, Jin CS, Yuan HJ and Cheng JZ: Long non-coding RNA HNF1A-AS is upregulated and promotes cell proliferation and metastasis in nasopharyngeal carcinoma. Cancer Biomark 16: 291-300, 2016.

18. Pan L, Liang W, Gu J, Zang X, Huang Z, Shi H, Chen J, Fu M, Zhang P, Xiao X, et al: Long noncoding RNA DANCR is activated by SALL4 and promotes the proliferation and invasion of gastric cancer cells. Oncotarget 9: 1915-1930, 2017.

19. Jiang N, Wang X, Xie X, Liao Y, Liu N, Liu J, Miao N, Shen J and Peng T: lncRNA DANCR promotes tumor progression and cancer stemness features in osteosarcoma by upregulating AXL via miR-33a-5p inhibition. Cancer Lett 405: 46-55, 2017.

20. Jin L, Fu H, Quan J, Pan X, He T, Hu J, Li Y, Li H, Yang Y, Ye J, et al: Overexpression of long non-coding RNA differentiation antagonizing non-protein coding RNA inhibits the proliferation, migration and invasion and promotes apoptosis of renal cell carcinoma. Mol Med Rep 16: 4463-4468, 2017.

21. Jia J, Li F, Tang XS, Xu S, Gao Y, Shi Q, Guo W, Wang X, He D and Guo P: Long noncoding RNA DANCR promotes invasion of prostate cancer through epigenetically silencing expression of TIMP2/3. Oncotarget 7: 37868-37881, 2016.

22. Salmena L: PTEN: History of a tumor suppressor. Methods Mol Biol 1388: 3-11, 2016.

23. Gao Q, Tang L, Wu L, Li K, Wang H, Li W, Wu J, Li M, Wang S and Zhao L: LASP1 promotes nasopharyngeal carcinoma progression through negatively regulation of the tumor suppressor PTEN. Cell Death Dis 9: 393, 2018.

24. Carnero A, Blanco-Aparicio C, Renner O, Link W and Leal JF: The PTEN/PI3K/AKT signalling pathway in cancer, therapeutic implications. Curr Cancer Drug Targets 8: 187-198, 2008.

25. Song LB, Li J, Liao WT, Feng Y, Yu CP, Hu LJ, Kong QL, $\mathrm{Xu}$ LH, Zhang X, Liu WL, et al: The polycomb group protein Bmi-1 represses the tumor suppressor PTEN and induces epithelial-mesenchymal transition in human nasopharyngeal epithelial cells. J Clin Invest 119: 3626-3636, 2009.

26. Edlich F: BCL-2 proteins and apoptosis: Recent insights and unknowns. Biochem Biophys Res Commun 500: 26-34, 2018. 